\title{
Frequency Map for the Human Cochlear Spiral Ganglion: Implications for Cochlear Implants
}

\author{
Olga Stakhovskaya, Divya Sridhar, Ben H. Bonham, and Patricia A. Leake \\ Epstein Laboratory, Department of Otolaryngology—Head and Neck Surgery, University of California San Francisco, 533 \\ Parnassus Ave., Room U490, San Francisco, CA 94143-0526, USA
}

Received: 6 September 2006; Accepted: 20 January 2007; Online publication: 21 February 2007

\begin{abstract}
The goals of this study were to derive a frequencyposition function for the human cochlear spiral ganglion (SG) to correlate represented frequency along the organ of Corti (OC) to location along the SG, to determine the range of individual variability, and to calculate an "average" frequency map (based on the trajectories of the dendrites of the SG cells). For both OC and SG frequency maps, a potentially important limitation is that accurate estimates of cochlear place frequency based upon the Greenwood function require knowledge of the total OC or SG length, which cannot be determined in most temporal bone and imaging studies. Therefore, an additional goal of this study was to evaluate a simple metric, basal coil diameter that might be utilized to estimate OC and SG length. Cadaver cochleae $(n=9)$ were fixed $<24 \mathrm{~h}$ postmortem, stained with osmium tetroxide, microdissected, decalcified briefly, embedded in epoxy resin, and examined in surface preparations. In digital images, the $\mathrm{OC}$ and $\mathrm{SG}$ were measured, and the radial nerve fiber trajectories were traced to define a series of frequency-matched coordinates along the two structures. Images of the cochlear turns were reconstructed and measurements of basal turn diameter were made and correlated with OC and SG measurements. The data obtained provide a mathematical function for relating represented frequency along the OC to that of the SG.
\end{abstract}

Correspondence to: Olga Stakhovskaya • Epstein Laboratory • UCSF Department of Otolaryngology-Head and Neck Surgery • 533 Parnassus Ave., Room U490, San Francisco, CA 94143-0526, USA. Telephone: +1-415-5024880; fax: +1-415-4762169; email: ostakhovskaya@ohns.ucsf.edu
Results showed that whereas the distance along the OC that corresponds to a critical bandwidth is assumed to be constant throughout the cochlea, estimated critical band distance in the SG varies significantly along the spiral. Additional findings suggest that measurements of basal coil diameter in preoperative images may allow prediction of $\mathrm{OC} / \mathrm{SG}$ length and estimation of the insertion depth required to reach specific angles of rotation and frequencies. Results also indicate that OC and SG percentage length expressed as a function of rotation angle from the round window is fairly constant across subjects. The implications of these findings for the design and surgical insertion of cochlear implants are discussed.

Keywords: critical bands, basal turn diameter

\section{INTRODUCTION}

Approximately 100,000 hearing-impaired individuals worldwide now have received cochlear implants (CI), and contemporary devices clearly provide remarkable benefit to many implant recipients. Considerable progress in the design of implantable electronics and speech processors over the last 20 years have allowed higher rates of stimulation and greater programming flexibility, contributing to higher overall levels of CI performance. Given that the larger numbers of channels available with the latest CI technology (e.g., "virtual channels") may allow more flexibility in the frequency ranges delivered to different cochlear regions, further improvement of CI performance may depend on optimizing the tonotopic mapping of individual channels relative 
to the actual position of stimulation sites in the cochlea. A number of recent studies have been directed toward providing a better understanding of the role of electrode location and spacing on various perceptual attributes of hearing with a CI (Blamey et al. 1996; Ketten et al. 1998; Pfingst et al. 2001; Skinner et al. 2002; Yukawa et al. 2004; Baumann and Nobbe 2006; Boex et al. 2006). Several psychophysical studies have shown that spectral distortions such as apical or basal shift (Dorman et al. 1997; Fu and Shannon 1999), nonlinear warping (Shannon et al. 1998), and compression or expansion of the applied frequency map (Baskent and Shannon 2003, 2004) decrease speech perception, thus suggesting that an optimum fit of the frequency map for a given electrode position may significantly improve the performance of the CI listener. Although some studies have shown that speech perception with a frequency-shifted implant map can substantially improve with auditory training and if the patient has more time to adapt to the map (Rosen et al. 1999; Fu et al. 2005), an initial optimum frequency fit may provide immediate benefit for implant recipients and a better basis for the subsequent adaptation that occurs in most CI users.

In most studies examining electrode position, Greenwood's frequency-position function (Greenwood 1961, 1990) has been used to estimate the characteristic frequencies for CI electrodes. This function was originally derived from frequency resolution-integration estimates (critical bandwidths) in humans and allows the estimation of represented frequencies along the organ of Corti (OC) as a function of percentage length. A potentially important consideration is that Greenwood's equation may provide an accurate estimate of frequency for CI electrodes only if the site of spike initiation in electrical excitation of the spiral ganglion (SG) neurons is near the OC, i.e., at the first node of Ranvier on the radial nerve fibers. However, radial nerve fibers begin to degenerate soon after the OC is damaged (Otte et al. 1978; Hinojosa et al. 1983; Leake and Hradek 1988; Nadol et al. 1989; Nadol 1990; McFadden et al. 2004). In contrast, SG cell somata degenerate relatively slowly in humans, and significant populations often survive even after decades of deafness, and/or after many years of CI use, even when radial nerve fibers are largely absent (Fayad et al. 1991; Nadol 1997; Khan et al. 2005). For these reasons, some contemporary "perimodiolar" electrode arrays (e.g., the Contour ${ }^{\mathrm{TM}}$ electrode from Cochlear Limited, Sydney, Australia, and the HiFocus ${ }^{\mathrm{TM}}$ with positioner and new Helix ${ }^{\mathrm{TM}}$ electrodes from Advanced Bionics, Sylmar, CA, USA) have been designed to place stimulating electrodes in close proximity to the modiolus to directly excite the SG cell somata within Rosenthal's canal.

A review of basic cochlear anatomy makes apparent the potential inaccuracy of assuming that the SG frequency map corresponds closely to that of adjacent OC. In the basal coil, the nerve bundles from the $\mathrm{OC}$ take a relatively direct radial course into the modiolus, but in the middle and apical turns, the trajectory of the fibers deviates significantly because Rosenthal's canal is much shorter than the OC and does not extend to the apical turn (Bredberg 1968; Glueckert et al. 2005). Thus, represented frequency along the SG must be offset from that of the OC. The relationship between $\mathrm{OC}$ and $\mathrm{SG}$ frequency maps also must change in different coils due to the change in radius of curvature along the spiral. It has been reported previously that Rosenthal's canal is not linearly related to the OC (Kawano et al. 1996), but the relationship between corresponding frequencymatched points along the OC and SG has never been studied.

Another potentially important limitation for CI studies is that accurate estimates of cochlear place frequency based upon Greenwood's function require knowledge of the total OC length, which cannot be determined in most temporal bone and imaging studies. Estimates of frequency based upon the average OC length may be inaccurate due to substantial individual variability.

The goals of this study were to evaluate a simple metric that might be utilized to estimate OC and SG length, to derive an accurate frequency-position function for the human SG, and to explore the implications of the differences between SG and OC frequency maps with respect to the design and surgical insertion of CIs. The data presented here should provide a basis for more accurate frequency estimates for implanted CI electrodes and should also be useful for computer modeling studies of the electrically stimulated cochlea.

Some preliminary data on the frequency-position map for the SG from a subset of the cochlear specimens included in this study were reported previously in the proceedings of the First International Electro-Acoustic Workshop (Sridhar et al. 2006).

\section{METHODS}

\section{Preparation of temporal bones}

Temporal bones $(n=9)$ were obtained from the Department of Pathology at the University of California San Francisco or from Life Legacy Foundation, a nonprofit tissue and organ bank for medical research. Specimens were harvested within $24 \mathrm{~h}$ after death or less and fixed by immersion in $10 \%$ phosphate-buffered formalin. The tympanic membrane was opened as soon as possible after removal of the specimen and the cochlea was gently perfused through the round and oval windows to facilitate access of fixative to the labyrinth. Mean age at 
death was 64 years with a range of 49 to 78 years. Six specimens from males and three from females (Table 1) were included in this study. Only one cochlea (left or right) was studied from each cadaver to better assess intersubject variability.

After fixation, the specimens were placed in $0.1 \mathrm{M}$ phosphate buffer and grossly dissected to isolate the petrous temporal bone. The otic capsule was thinned with a diamond burr to orient the cochlea and to define ("blue line") the cochlear turns. The specimens were postfixed in $1 \%$ osmium tetroxide in 0.1 $\mathrm{M}$ phosphate buffer ( $\mathrm{pH} 7.4$ ) with $1.5 \%$ potassium ferricyanide added (to enhance contrast) for $4 \mathrm{~h}$ to stain the radial nerve fibers. Next, the otic capsule bone above each coil was further thinned, and then completely removed by microdissection to expose the vestibule and scala vestibuli of each turn and to provide an unobstructed view of the entire length of osseous spiral lamina and associated OC, spiral ligament, and stria vascularis. To relate anatomical data to images of temporal bones that can be obtained in living subjects, the center of the vestibule (V) was chosen as an anatomic landmark that could be defined in x-ray or computerized tomography (CT) techniques. A marker was created by cutting a small notch in the stria vascularis of the upper basal turn at the point nearest to $\mathrm{V}$. The marker was maintained throughout subsequent histological processing to define the position of this landmark in the final cochlear reconstruction. The specimens were then decalcified briefly $(24-36 \mathrm{~h}$ ) in $0.2 \mathrm{M} \mathrm{EDTA} \mathrm{to}$ fully visualize the radial nerve fibers. Finally, the cochlea was dehydrated gradually in ethanol and embedded in epoxy resin (Epo-Tek 301, Epoxy Technology, Billerica, MA, USA).

\section{Cochlear surface preparations}

Following embedding, each cochlea was bisected through the middle of the modiolus, in a plane that was oriented as parallel as possible to the radial nerve fibers on each side of the basal turn. Each half-coil of the cochlea was then isolated from the two larger blocks (using razor blades to cut the plastic after the blocks were warmed to about $100^{\circ} \mathrm{C}$ ) and remounted on a glass slide in a classic surface preparation. However, because the OC and SG of the human cochlea are vertically offset from one another, the OC was positioned upside down on the slide (with the reticular lamina closer to the slide and the scala tympani aspect of the basilar membrane facing upward) so that the OC was oriented flat and parallel to the slide, and the adjacent SG was offset above the OC. The hook region, where the basilar membrane and OC arch around the round window, was cut into three or more pieces to allow the OC to be mounted flat and parallel to the slide. Also, in the apex it was necessary to divide the half coils into two pieces so the OC would lie flat on the slide, which was important for reducing parallax and ensuring accurate measurements.

\section{Measurements of the OC and SG: charting radial} nerve fiber trajectories

Each piece of the surface preparation was examined in the light microscope (Zeiss Axioskop 2, Oberkochen, Germany), and high-resolution digital images $(2,584 \times 1,936$ pixels $)$ were captured using Photoshop 5.0, a Kodak (Rochester, NY, USA) digital camera, and a personal computer. For calibration, an image of a micrometer scale was captured and superimposed on each image to be evaluated. In Canvas 8 software, the center of the OC in each image was traced along the tops of the pillar cells and measured in each segment of the surface preparation (Fig. 1). Small sectors (0.5 $\mathrm{mm}$ ) of the $\mathrm{OC}$ and associated SG were then removed from the surface preparation by heating the slide to about $100^{\circ} \mathrm{C}$ on a hot plate and making razor blade cuts parallel to the radial nerve fibers at each of 10-12 locations throughout the cochlear spiral. These pieces were remounted on epoxy blanks, and semithin sections $(5 \mu \mathrm{m})$ were cut in the radial

TABLE 1

\begin{tabular}{lllll}
\hline \multicolumn{5}{c}{ TABLE 1 } \\
\hline & Source, & & \\
\hline Specimen \# & Source & Postmortem fixation within $(h)$ & Age & \\
\hline 4 right & UCSF & 13 & 65 & Gender \\
5 right & LLF & 8 & 78 & Male \\
6 right & UCSF & 22 & 64 & Male \\
7 right & UCSF & 8 & 53 & Female \\
8 right & LLF & 4 & 60 & Male \\
13 left & LLF & 2 & 59 & Male \\
14 right & UCSF & 17 & 72 & Female \\
15 left & UCSF & 24 & 49 & Male \\
16 right & UCSF & 23 & Male & Female \\
\hline
\end{tabular}

UCSF $=$ Department of Pathology at the University of California San Francisco, LLF = Life Legacy Foundation 
plane and stained with Toluidine blue (Fig. 2). The blocks containing the most basal and apical segments of the SG were sectioned serially, and the number of sections to reach the terminus of the SG was counted to determine the precise length of the SG (i.e., number of sections from the beginning of the block to the end of Rosenthal's canal at the base and apex multiplied by section thickness). Sections were collected in other locations to examine SG morphology. Two additional measurements were made in the radial sections. Specifically, the distances from the OC to the center of the SG and to the modiolar wall (MW) adjacent to the center of the SG (ideal position of a perimodiolar CI electrode) were measured at each location where the radial nerve fiber trajectories were traced. These values were plotted as a series of points on the digital images of the surface preparations (Fig. 1). This allowed us to draw a line representing the center of the SG and a second line representing the innermost radius of the MW adjacent to the SG by interpolating between two sets of points and following the curvature of the modiolar bone in the images of each piece of the surface preparation. The lengths of the SG and adjacent MW were determined by measuring the two lines in each image and summing the measurements for all the individual segments. One cochlea (\#9) was damaged at the base such that the terminus of the SG area could not be determined, and data were obtained only for basilar membrane measurements.

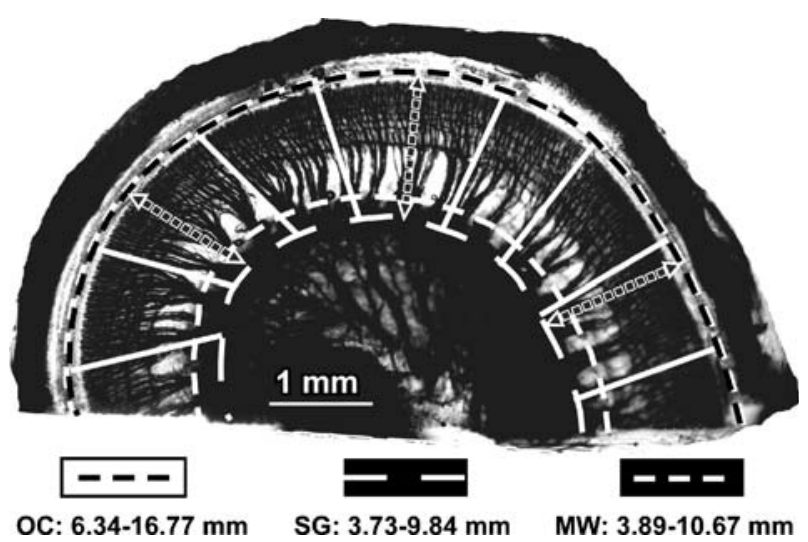

FIG. 1. Digital image of the lower basal turn, taken from a surface preparation of an epoxy-embedded human cochlea. The black dashed line defines the OC length, which was $10.43 \mathrm{~mm}$ in this example. The dotted radial lines indicate where radial sections were prepared to examine the SG and to measure the distances from the OC to the SG and MW. White dashed lines represent the SG (inner line), which measured $6.11 \mathrm{~mm}$ at the approximate center of Rosenthal's canal (as estimated in the radial sections), and the MW (outer line) adjacent to the SG, which measured $6.78 \mathrm{~mm}$ for this specimen. The white lines show the tracings of radial nerve fiber trajectories that were used to define the frequency-matched coordinates on the OC and SG. Scale bar, $1 \mathrm{~mm}$. Modified from Sridhar et al. (2006) and reproduced from S. Karger AG, Basel.

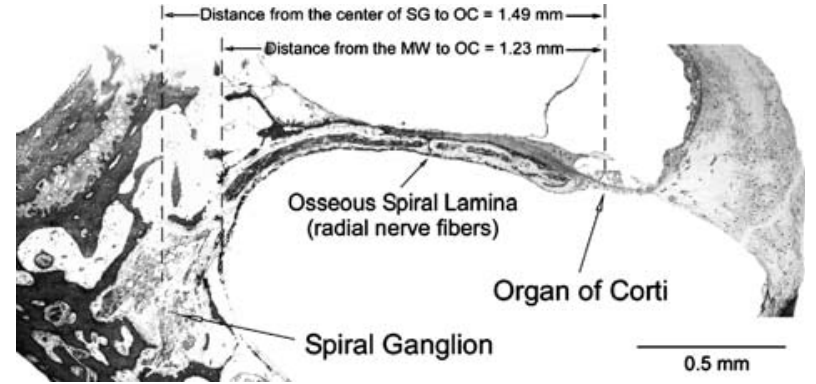

FIG. 2. Digital image of a radial section containing both the OC and SG. This section is taken from the lower basal turn, approximately $20 \%$ from the basal end of the OC. The radial distances from the OC to the center of the SG $(1.49 \mathrm{~mm})$ and from the OC to the MW $(1.23 \mathrm{~mm})$ were measured at several points and plotted on the surface preparation images. Scale bar, $0.5 \mathrm{~mm}$.

As illustrated in Figure 1, the radial nerve fibers were clearly visible in the surface preparations after decalcification of the osseous spiral lamina and staining the neurons black with osmium tetroxide. Individual fascicles were easily identified throughout the spiral of each cochlea. This allowed us to trace the trajectories of the radial nerve fibers from the OC to the associated SG region in the digital images and thus to define a series of frequency-matched points along the $\mathrm{OC}$ and $\mathrm{SG}$ in each cochlea. After calibrating the images, the distances between these series of points along each of the two structures were determined and expressed as percentage of total length.

\section{Measurements of the cochlear basal turn diameters}

Digital images from all the individual segments of the cochlear surface preparations were assembled graphically, by digitally apposing and best-fitting the images of all the individual sectors in Canvas 8 software, to reconstruct the course of the OC and SG. The maximum diameter of the basal turn was measured in the reconstructed images by measuring a line drawn from the OC at the estimated location of the center of the round window, through the modiolus (center of the cochlear spiral) to the opposite side of the basal coil. A second measurement of basal turn diameter was also made, orthogonal to the first, as illustrated in the schematic diagram in Figure 3.

\section{Calculations of characteristic frequencies vs angle of rotation for the OC and SG in reconstructed images}

To calculate the characteristic frequencies of the OC and SG as a function of the angle of rotation from the round window, we used the same reconstructed OC and SG images prepared as described above. An artificial grid was superimposed over the image for 


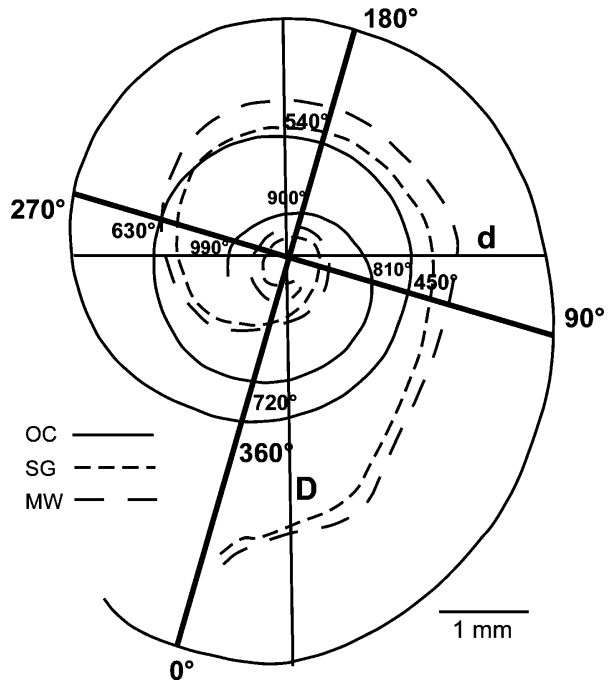

FIG. 3. Schematic drawing of a reconstruction of the OC and SG in one of the cochlear surface preparations illustrating the two basal turn diameter measurements. The maximum diameter of the basal coil was measured from the approximate center of the round window $(D)$, and a second diameter $(d)$ was measured orthogonal to the first. For making angular measurements of insertion depths and frequency range, a grid was superimposed on the reconstructed cochlea with a reference point $0^{\circ}$ at $1 \mathrm{~mm}$ from the basal end of OC. In this example, the OC extends slightly beyond $990^{\circ}$, whereas SG terminates at approximately $720^{\circ}$ from the round window.

each cochlea as illustrated in Figure 3. Organ of Corti and SG lengths were measured at intersections with the grid at intervals of $45^{\circ}$ of rotation. The reference point ( 0 degrees) was chosen as $1 \mathrm{~mm}$ from the basal end of the OC. This point was chosen because the initial portion of the OC curves inferiorly and would project as a continuous zero or close-to-zero degree value on the grid and also would be extremely difficult to define in imaging studies. We estimated that $1 \mathrm{~mm}$ from the basal end of the OC would represent the approximate position of the anterior margin of the round window, which could be used as a landmark reference point in imaging studies. OC and SG lengths measured at each $45^{\circ}$ interval of rotation were expressed in millimeters to estimate the depth of insertion of a CI electrode necessary to reach this point. Data were also expressed as percentage distance to calculate frequency vs rotation angle using Greenwood's equation (Greenwood 1990). Greenwood's equation for frequency distribution along the OC is:

$$
F=A^{*} 10^{a x}-k
$$

where $F$ is frequency in Hertz and $x$ is the position on the basilar membrane re the apex and can be expressed in millimeters, proportion ( 0 to 1$)$, or percent distance of the total length. Greenwood provided coefficients for humans of $A=165.4, a=2.1$ (if $x$ is expressed as proportion of total basilar membrane length), and $k=0.88$ (to give a lower frequency limit of $20 \mathrm{~Hz}$ ). These values were used here to calculate frequencies for the OC and SG at $45^{\circ}$ intervals along the spiral in individual cochleae. Because Greenwood's equation was applied for individual cochleae using the above-mentioned constants and percent distance of basilar membrane length as $x$, the upper and lower frequency limits were set at $20-20,677 \mathrm{~Hz}$ for all the cochleae. To define the frequencies for the SG, the radial fibers were traced from the SG at the intersection of the grid line for each $45^{\circ}$ interval to the associated point on the OC. The characteristic frequencies were then defined from the OC percent distance value using Greenwood's equation.

\section{RESULTS}

\section{OC and SG length}

As shown in Table 2, the mean length of the OC for nine cochlear specimens measured in the reconstructed digital images was $33.13 \mathrm{~mm}( \pm 2.11 \mathrm{~mm}$, SD). Spiral ganglion length was substantially shorter and averaged only $13.69 \mathrm{~mm}( \pm 0.80 \mathrm{~mm}, \mathrm{SD})$. Direct morphometric analyses showed that the basal $2.35 \%$ and apical $10.72 \%$ of the OC extended beyond the end of the SG, so that no SG cell bodies were positioned directly radial to these sectors of the OC. In spite of the great intersubject variability in OC and SG lengths, the ratio of SG to OC length was fairly similar in different-sized cochleae, with a range of 0.40 to 0.43 (Table 2). The data showed good correlation $\left(R^{2}=0.76\right)$ between the total lengths of the OC and SG for these cochleae as presented in Figure 4. The total length of the MW directly adjacent to the SG was also measured as an estimate of the insertion distance required to ideally position a perimodilar CI array against the modiolus. This length averaged $15.49 \mathrm{~mm}( \pm 0.69 \mathrm{~mm}, \mathrm{SD})$, a value that was only slightly greater than the length of the SG measured at the approximate center of Rosenthal's canal, with a mean difference between the two measurements of $1.81 \mathrm{~mm}$. The ratio of MW to OC length ranged from 0.44 to 0.49 , with a mean value of 0.47 .

\section{SG frequency-position function}

Tracing the radial nerve fibers from the OC to the corresponding SG region defined a set of frequencymatched coordinates along the OC and SG. The data were expressed as percent of the distance from the base of the cochlea for the OC and the SG (at its approximate anatomical center as defined in the 
TABLE 2

\begin{tabular}{|c|c|c|c|c|c|c|c|c|c|c|}
\hline \multicolumn{11}{|c|}{ Anatomical measurements collected for nine specimens in the study } \\
\hline Specimen & $4 R$ & $5 R$ & $6 R$ & $7 R$ & $8 R$ & $13 L$ & $14 R$ & $15 L$ & $16 R$ & Mean $\pm S D$ \\
\hline OC length (mm) & 32.31 & 33.44 & 31.41 & 30.5 & 36.16 & 33.09 & 36.87 & 32.45 & 31.95 & $33.13 \pm 2.11$ \\
\hline SG length (mm) & 14 & 14.2 & NA & 12.54 & 14.58 & 13.41 & 14.62 & 13.44 & 12.7 & $13.69 \pm 0.8$ \\
\hline SG/OC length & 0.43 & 0.42 & NA & 0.41 & 0.4 & 0.41 & 0.4 & 0.41 & 0.4 & $0.41 \pm 0.01$ \\
\hline MW length (mm) & 15.79 & 15.9 & NA & 14.35 & 16.24 & 15.2 & 16.33 & 15.15 & 14.97 & $15.49 \pm 0.69$ \\
\hline MW vs SG difference & 1.79 & 1.7 & NA & 1.81 & 1.66 & 1.79 & 1.71 & 1.71 & 2.27 & $1.81 \pm 0.2$ \\
\hline$\%$ OC basal w/o SG & 1.98 & 3.47 & NA & 1.31 & 0.4 & 3.41 & 1.84 & 2.13 & 3.29 & $2.35 \pm 0.9$ \\
\hline$\%$ OC apical w/o SG & 9.22 & 11.33 & NA & 11.64 & 10.26 & 9.4 & 12.99 & 11.37 & 9.58 & $10.72 \pm 1.33$ \\
\hline$\%$ OC length at $\mathrm{V}$ & 59.36 & 64.74 & 60.01 & 59.08 & 55.56 & 59.56 & $\mathrm{NA}$ & 61.14 & 60.31 & $59.97 \pm 2.54$ \\
\hline Frequency at $\mathrm{V}(\mathrm{Hz})$ & 1,035 & 764 & 998 & 1,051 & 1,273 & 1,023 & NA & 937 & 982 & $1,000 \pm 140$ \\
\hline
\end{tabular}

Data collected in nine cochleae indicate relatively large intersubject variability in OC and SG lengths, but a fairly consistent ratio between the lengths of the two structures. The length of the MW directly adjacent to the SG differed from the SG length measured at the center of Rosenthal's canal by less than 2 mm. Dissection error resulted in damage to the basal SG in specimen $6 \mathrm{R}$ and precluded measuring SG length. The marker for $\mathrm{V}$ was lost during the processing of the cochlea and could not be identified in specimen $14 \mathrm{R}$.

radial sections). Percentage length along the SG was found to relate consistently and predictably to percentage length along the OC, with minimal intrasubject and intersubject variability (Fig. 5a).

In our earlier preliminary report that included measurements from six cochlear specimens, the data were fit with a third-order polynomial function. Because it is logical to assume that our normalized data should cover the region from 0 to $100 \%$ of both the OC and SG, that polynomial function was constrained to begin at the point $x=0, y=0$, to end at $x=100, y=100$, and to be monotonic (Sridhar et al. 2006). The addition of two more cochlear specimens to the data set did not eliminate a systematic bias observed near the base (up to 20\%) for the thirdorder polynomial, so the final set of data in this report was fit by a different mathematical function:

$$
y_{\mathrm{est}}(x)=\frac{100}{1+\left(A \frac{100}{x}+B \frac{x}{100}-A-B\right)^{2}}
$$

To model the frequency-matched coordinates along the SG, $x$ is the percent distance from the base of the OC and $y_{\text {est }}$ is the percentage distance of the corresponding location along the SG. This function increases monotonically over the data range, and passes through the two endpoints $(0,0)$ and $(100,100)$. Varying parameters $A$ and $B$, while performing a least-squared-error optimization, resulted in the values $A=0.22$ and $B=-0.93$, and a high $R^{2}$ value $(0.9978)$. The mean residual error, $y_{\text {meas }}-y_{\text {est }}$, of $0.04 \%$ indicated a small bias in the position estimate provided by the equation. Initial visual inspection indicated that the fit error was not systematic, and a runs-test on the signs of the residual errors failed to reject the null hypothesis that the observed sequence was random $(p>0.05)$.
As mentioned previously, we also measured a comparable series of frequency-matched coordinates between the $\mathrm{OC}$ and the MW at the point closest to the anatomical center of the SG. These measurements were intended to approximate the course of an optimally positioned perimodiolar CI array and may provide a basis for increasing the accuracy of estimates of frequency and optimum insertion distances for specific arrays. The data representing percent distance along the $\mathrm{OC}$ and frequency-matched distances along the MW adjacent to the SG are shown in Figure 5b. The data for this set of measurements can also be approximated by the function in Equation 1, where $x$ is again the percent distance from the base of the OC, but $y_{\text {est }}$ is now the percentage distance of the corresponding position along the MW directly adjacent to the SG. In this case, optimization resulted in parameter values $A=0.23$ and $B=-0.99$, with resulting $R^{2}=0.9977$.

The nerve fibers in the region from about 20 to $60 \%$ of the OC length take a radial trajectory. The

\section{Correlation Between SG Length and OC Length}

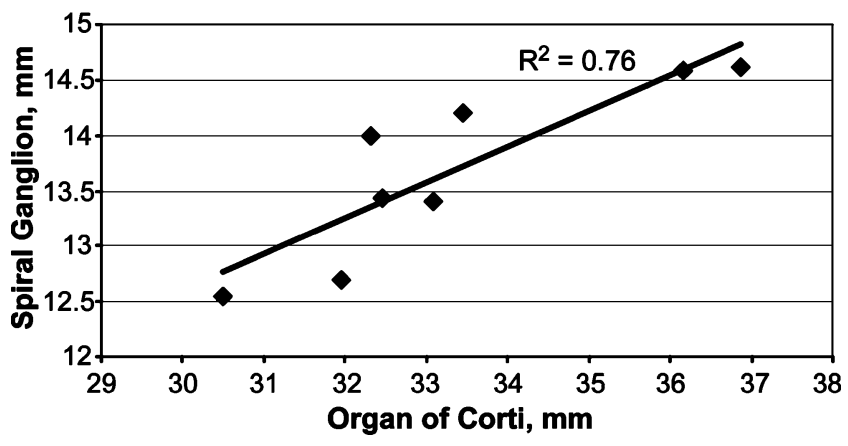

FIG. 4. A significant correlation is shown between total length of OC and SG in our data from eight human temporal bones $(n=8)$. 
a Spiral Ganglion vs Organ of Corti Percentage Length

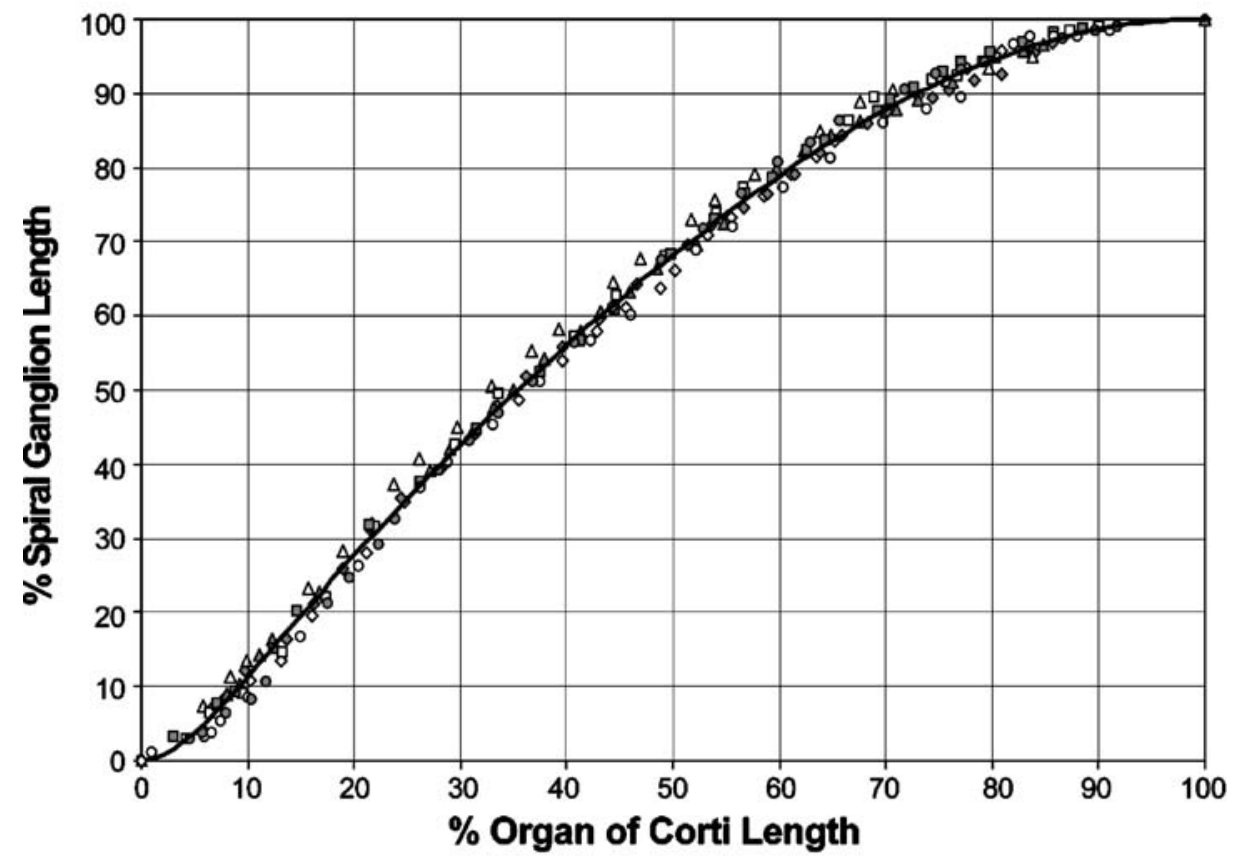

b Modiolar Wall vs Organ of Corti Percentage Length

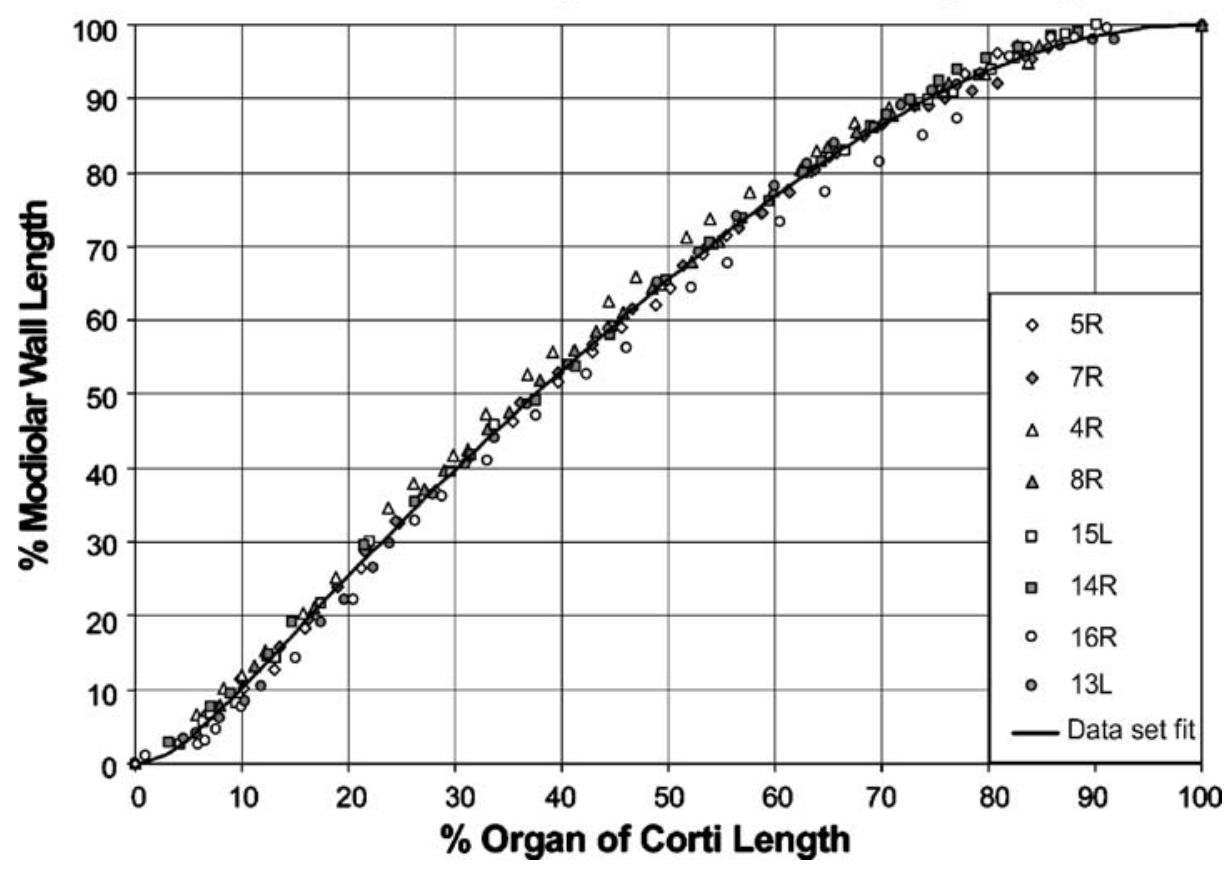

FIG. 5. The frequency-matched positions along the SG and OC (a), and along the MW adjacent to the SG (b), are plotted for eight individual cochlear specimens. Percentage length along the SG and MW were found to relate consistently and predictably to percentage length along the OC, with minimal intersubject variability.

mean distance from the base of the $\mathrm{OC}$ where the fibers begin to exhibit a more tangential course toward the associated region of the SG was $62 \%$ $( \pm 3.2 \%, \mathrm{SD})$ (approximately $\left.360^{\circ}\right)$ from the base of the OC, which corresponds to about $81 \%$ of the total SG length $( \pm 3.1 \%, \mathrm{SD})$.
Estimates of critical band distance in the human SG

The frequency-position map for the SG, and for the MW adjacent to the SG, are computed by combining the above equations with Greenwood's frequency- 
position function for the OC. Computing this map allows us to illustrate the SG's compressive distortion of the OC frequency map by converting a template of constant distances on the OC to the corresponding distances along the SG. The OC template is composed of contiguous critical band distances, which, by a well-supported hypothesis, appear to be constant distances along the basilar membrane. Although critical bandwidth estimates have varied (with psychophysical task, method of measurement, stimulus level, and subjects and their ages), the bandwidths within different data sets still correspond to constant distances, although different ones, which remain in the neighborhood of $1 \mathrm{~mm}$ (Greenwood 1961, 1991). A representative critical band distance of $1 \mathrm{~mm}$, given a conventional 35-mm basilar membrane, corresponds to $1 / 35$ th of the partition's length, or about $2.9 \%$ of the length of the OC in humans. This normalized schema, as noted earlier, imposes a constant frequency range on all cochleae and the same number of critical bands. For the average cochlea in our study, the OC was $33.13 \mathrm{~mm}$ long, and thus, the critical band distance would be 0.95 $\mathrm{mm}(1 / 35$ th or $2.9 \%$ of 33.13$)$. Because the critical band distance is constant over the length of the OC, the corresponding critical band distance along the SG can be computed by differentiating Equation 1:

$$
\begin{aligned}
y_{\text {est }}{ }^{\prime}(x) & =\frac{d y_{\text {est }}(x)}{d x} \\
& =\frac{-2\left(B-A\left(\frac{100}{x}\right)^{2}\right)\left(A \frac{100}{x}+B \frac{x}{100}-A-B\right)}{\left(1+\left(-A \frac{100}{x}-B \frac{x}{100}+A+B\right)^{2}\right)^{2}}
\end{aligned}
$$

where $x$ corresponds to percent distance along the OC, $y$ corresponds to percent distance along the SG, and parameters $A$ and $B$ are those determined by fitting Equation 1 to the SG measurements $(A=0.22$; $B=-0.93)$. The SG length, $\Delta y_{\mathrm{mm}}$, corresponding to one critical band distance along the $\mathrm{OC}$ at distance $x_{\mathrm{mm}}$ from the base, can be approximated by:

$$
\Delta y_{\mathrm{mm}} \approx 13.69 * y_{\text {est }}\left(x_{\mathrm{mm}} * 100 / 33.13\right) * 0.95 / 33.13
$$

The equivalent lengths of the SG regions corresponding to the critical band distances along the $\mathrm{OC}$ are shown in Figure 6. Unlike the OC critical band distances, which remain constant at $1 \mathrm{~mm}$ from base to apex, the associated distances along the SG vary as a function of position. In the hook region, SG critical bands rapidly reach maximum values at approximately $3-5 \mathrm{~mm}$ of OC distance and then become progressively narrower. Marked compression of the SG critical band distance was observed in the

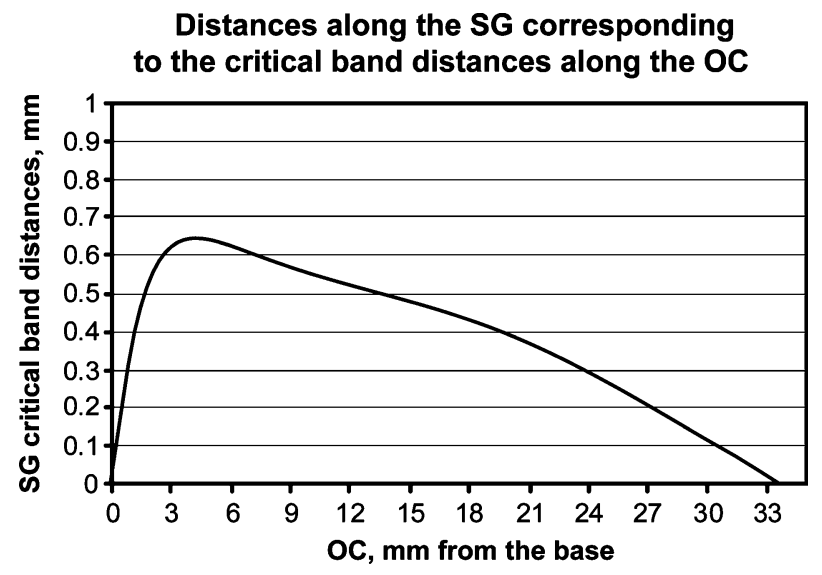

FIG. 6. Distances along the SG corresponding to critical band distances along the OC. Unlike critical band distances for the OC, which remain constant from base to apex, the associated SG critical band distances vary as a function of position.

apical $40 \%$ of the cochlea (apical to $\sim 21 \mathrm{~mm}$; frequencies of $\sim 1,000 \mathrm{~Hz}$ and lower), where the radial nerve fibers take an increasingly tangential course from the OC into the SG.

An anatomical reference point for relating the SG frequency map to living $\mathrm{Cl}$ subjects

via imaging studies

The landmark corresponding to $\mathrm{V}$ can be easily imaged in living subjects, and the CI electrode closest to $\mathrm{V}$ can be determined (see Fig. 10). In our cochlear specimens, the anatomical reference point $\mathrm{V}$ had a mean frequency of $1,000 \mathrm{~Hz}$, with very tight data scatter for six out of the eight cochleae, whereas the remaining two cochleae differed significantly from the average data, yielding quite a wide range of represented frequency from 1,275 to $760 \mathrm{~Hz}$ at the point $\mathrm{V}$ (Table 2). No significant correlation with OC or SG length was found.

\section{Basal turn diameter measurements}

The diameter of the basal turn can be measured in imaging studies of living CI recipients, and it has been reported that such data can be applied to predict insertion depth angles for CI electrodes (Escude et al. 2006). Therefore, we made comparable measurements in our anatomical specimens. The values measured for the maximum diameter at the approximate center of the round window ranged from 6.9 to $8.2 \mathrm{~mm}$. Moreover, the diameter was significantly correlated with total basilar membrane length $\left(R^{2}=0.77\right)$ and total SG length $\left(R^{2}=0.88\right)$, as illustrated in Figure 7a, b. Measurements of a second 
a

Correlation between OC Length and Cochlear Diameter

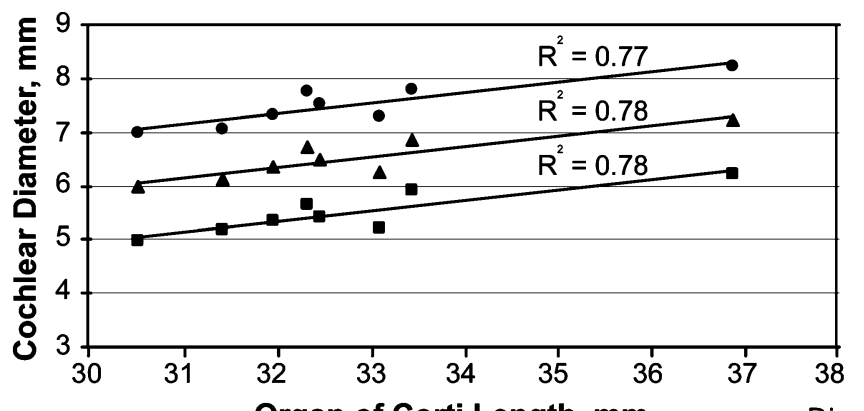

Organ of Corti Length, $\mathbf{m m} \quad \bullet$ Diameter at RW

$\triangle$ Mean, 2 Diameters

- Orthogonal to Diameter at RW
Correlation between SG Length and Cochlear Diameter

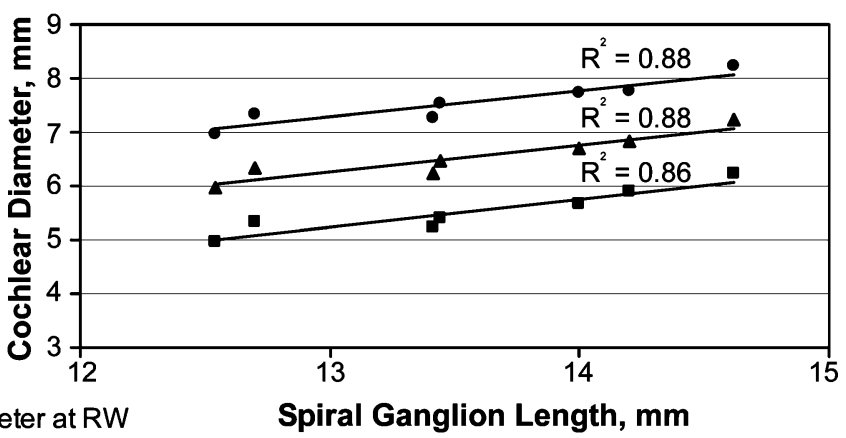

Spiral Ganglion Length, $\mathrm{mm}$

FIG. 7. In digital reconstructions of each cochlea, the maximum diameter of the basal turn was measured at the approximate center of the round window. A second measurement was made orthogonal to the first and the average of these two diameters was calculated. All three diameter measurements showed a significant correlation with overall OC length, as well as the length of the SG.

diameter, orthogonal to the first, showed an almost identical trend in correlation with OC and SG lengths.

In imaging studies, estimates of the basal turn diameter may be affected by the precise angle of view (Escude et al. 2006), especially in planar radiographic images. One way of limiting the impact of such inaccuracy would be to average the two diameters. Therefore, we also calculated the mean of the two diameters. These values also showed a strong correlation with total length of OC and SG.

Electrode insertion distances and SG and OC place frequencies vs angle of rotation

Absolute distances in millimeters required to reach specific angles of rotation along the OC or SG vary systematically with the overall length of these structures (Fig. 8). Because our measurements were made from the cochlear base, the difference between the shortest and longest cochleae in our set of specimens

OC and SG absolute distances vs. angle of rotation

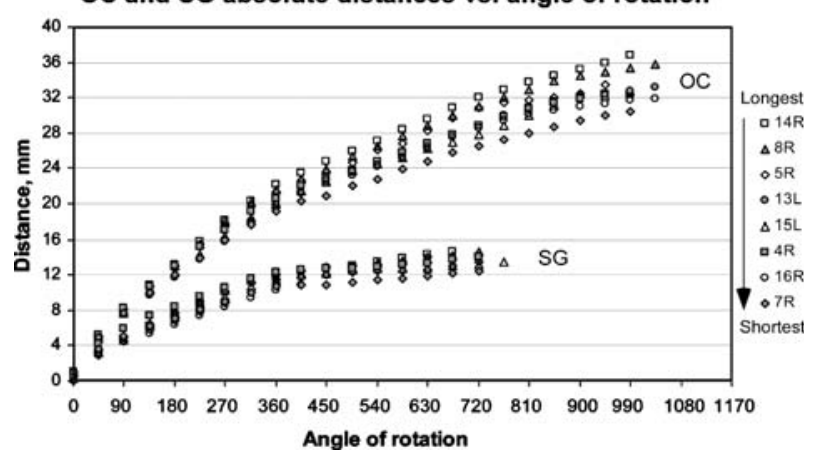

FIG. 8. Absolute distances along OC and SG measured at different angles of rotation from the round window for eight individual cochleae. Icons at the right of the graph are shown in order from the longest to the shortest OC. gradually increased from the base to the apex of the cochlea and, at the more apical rotational angles, reached 5-6 $\mathrm{mm}$.

The correlation between the total length of OC and the distance from the base becomes significant $(p<0.05)$ at a grid intersection angle of $225^{\circ}$ (Spearman rank order test) and reaches systematically higher values at more apical angles. The SG showed the same trend with a larger degree of individual variability. The correlation between the total length of SG and the distance required to reach a specific point along the SG becomes significant $(p<0.05)$ at an angle of $270^{\circ}$ relative to the superimposed grid (Spearman rank order test).

Measurements of the percentage distance of the $\mathrm{OC}$ and SG lengths required to reach specific angles of rotation along the cochlea (Fig. 9) show considerably less intersubject variability as compared to the absolute values in millimeters shown in the previous figure. Because percent distance of OC is

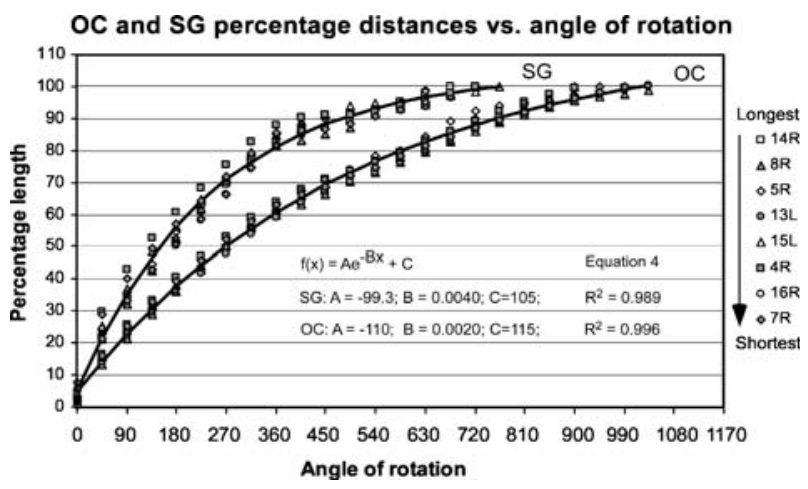

FIG. 9. Percentage length of OC and SG as measured at different angles of rotation from the round window. 
TABLE 3

Frequencies along the OC and SG at different angles of rotation

\begin{tabular}{|c|c|c|c|c|}
\hline $\begin{array}{l}\text { Angle of rotation } \\
\text { from round window }\end{array}$ & OC mean freq & OC freq range & $S G$ mean freq & SG freq range \\
\hline $0^{\circ}$ & 17,857 & $17,623-18,120$ & 17,225 & $14,734-20,677$ \\
\hline $90^{\circ}$ & 6,501 & $5,867-7,253$ & 6,193 & $5,111-7,132$ \\
\hline $180^{\circ}$ & 3,239 & $2,812-3,567$ & 3,174 & $2,700-3,496$ \\
\hline $270^{\circ}$ & 1,659 & $1,460-1,906$ & 1,539 & $1,381-1,832$ \\
\hline $360^{\circ}$ & 920 & 809-1,063 & 785 & $676-916$ \\
\hline $450^{\circ}$ & 601 & $531-699$ & 550 & $444-699$ \\
\hline $540^{\circ}$ & 407 & $331-462$ & 366 & $283-400$ \\
\hline $630^{\circ}$ & 263 & $208-302$ & 215 & $170-262$ \\
\hline $720^{\circ}$ & 152 & $93-184$ & 58 & $20-172$ \\
\hline $810^{\circ}$ & 86 & $63-108$ & & \\
\hline $900^{\circ}$ & 44 & 20-61 & & \\
\hline $990^{\circ}$ & 27 & 20-39 & & \\
\hline
\end{tabular}

correlated to the represented frequency (by Greenwood's equation), the limited variability in these data indicates that frequency vs angle of rotation estimates may be relatively constant in different-sized cochleae. The data describing the relationships between distance along OC or SG expressed as a percent of total length and angular position around the cochlear spiral were best fit by the exponential function presented on the plot. This equation provides the convenience of a mathematical expression for averaged data when a direct measurement of OC or SG length is problematic. Thus, it is possible to estimate the percentage length of OC or SG by substituting angular location of the electrode contacts into the equations.

Table 3 shows the mean frequencies for the OC and SG and the ranges calculated for individual cochlear specimens for our data set for $90^{\circ}$ increments in angle of rotation from the round window. Frequency calculations for the OC are based on Greenwood's equation. To determine the frequencies for the SG, the radial fibers were traced from the specific points on SG defined by the grid intersections out to the associated region on the $\mathrm{OC}$.

\section{DISCUSSION}

Measurements of OC, SG, and MW and implications for $\mathrm{Cl}$

Measurements of OC length in the present data set of nine cochleae showed substantial intersubject variability with a range from 30.5 to $36.87 \mathrm{~mm}$ (mean, 33.13 $\mathrm{mm}$ ). This finding is consistent with numerous prior reports indicating a large extent of variation in human cochlear length (Bredberg 1968; Ulehlova et al. 1987; Wright et al. 1987; Hardy 1968; Kawano et al. 1996; Ketten et al. 1998: Skinner et al. 2002). We did not observe a significant difference between female and male OC length as reported by Sato et al. (1991), who found OC lengths of $32.3 \pm 1.8 \mathrm{~mm}$ for females and $37.1 \pm 1.6 \mathrm{~mm}$ for males (total $n=18$ ). However, our findings are consistent with those of Ketten et al. (1998) and Skinner et al. (2002), who also reported no significant male-vs-female difference in $\mathrm{OC}$ length, based upon high-resolution CT studies and data from a large group of temporal bones $(n=33)$. The mean value for OC length $(33.13 \mathrm{~mm})$ in our specimens was very similar to the value $(33.4 \mathrm{~mm})$ obtained in the latter study.

Spiral ganglion length, measured in the center of Rosenthal's canal, was significantly shorter and ranged from only 40 to $43 \%$ of OC length, with absolute values of 12.54 to $14.62 \mathrm{~mm}$ (mean of 13.69 $\mathrm{mm})$. Our data showed a significant correlation between total length of OC and SG, which suggests that if OC length is known (e.g., from high-resolution CT images), it can be used to estimate the length of the SG. Kawano et al. (1996) reported a longer mean value of $15.98 \mathrm{~mm}$ for Rosenthal's canal in the human cochlea. The cochlear reconstructions in that study were made with a computer-aided 3D technique, and the authors also reported a systematic difference between the 2D and 3D reconstructions, with the larger values obtained with the 3D reconstructions. It should be noted that the surface preparation method used in our study applies a series of 2D measurements to evaluate a 3D structure, and we cannot rule out the possibility that it could introduce a slight but systematic inaccuracy in the data. However, carefully positioning the OC parallel to the slide minimized this possibility. The advantage of this technique was that it allowed us to make accurate direct measurements of the relevant structures in digital images without losing significant information, to directly trace the nerve fiber trajectories, and to remove selected regions for more detailed analysis (e.g., precise definition of the end of the $\mathrm{SG}$ ) in serial sections. 
Perimodiolar electrodes, such as the Cochlear Contour ${ }^{\mathrm{TM}}$ and Advanced Bionics Helix ${ }^{\mathrm{TM}}$, are designed to target the SG cell bodies directly (or their central axons), and it is obvious that such electrodes should be substantially shorter than an electrode positioned along the OC to cover the same frequency range. The distance along the outer wall of the modiolus directly adjacent to the SG differs from the length of the SG (center of Rosenthal's canal) by only about $1-2 \mathrm{~mm}$, with absolute values ranging from 14 to $17 \mathrm{~mm}$. These data suggest that the length of an implanted array necessary to approximate the entire length of the SG should not exceed about 17 $\mathrm{mm}$. At the same time, it is important to note that SG terminated at a rotation angle of $630-720^{\circ}$ in our specimens. Thus, although $17 \mathrm{~mm}$ is a sufficient length to reach the end of the ganglion, the insertion angle is the critical metric, and the depth of insertion required to achieve an ideal angle may vary considerably among individual subjects and different devices. Moreover, considering that the incidence of mispositioned electrodes is fairly high, averaging about 25-30\% (Skinner et al. 2002), and that longer insertions are more likely to cause trauma (Wardrop et al. 2005a, b), the issues of the length of electrode array and angle of insertion necessary to achieve the optimum results are potentially very important.

Other CI electrode designs are intended to be positioned along the OC to stimulate surviving radial nerve fibers (e.g., MedEl Combi-40). These electrodes will require longer insertion depths, and the frequency map is assumed to be similar to the OC. In individuals with more residual hearing and shorter durations of deafness, this type of design should theoretically be beneficial in increasing spectral selectivity (Hochmair et al. 2003). However, it is not clear whether stimulation of the radial nerve fibers is actually achieved with such electrodes, and it remains an open question as to where the spike initiation site(s) is/are with direct electrical activation and with various CI electrodes.

To illustrate this point, the mean location for our anatomical landmark $\mathrm{V}$ was $60 \%$ from the base of the OC, and based upon Figure 9, this distance corresponds to the $360^{\circ}$ of rotation. This suggests that a CI electrode carrier that takes a course under the OC would have to place a stimulating contact at a point 20 $\mathrm{mm}$ from the extreme base of the OC (i.e., $60 \%$ of $33.31 \mathrm{~mm}$ ), and at an angle of $360^{\circ}$, to position the electrode at $\mathrm{V}(1,000 \mathrm{~Hz})$ in the average cochlea. In contrast, for a perimodiolar CI electrode carrier such as the Cochlear Contour ${ }^{\mathrm{TM}}$ shown in Figure 10, the approximate insertion depth required to reach $\mathrm{V}$ $\left(360^{\circ}\right)$ would be only $\sim 11.5-12.5 \mathrm{~mm}$ ( $84 \%$ of 13.69 mm for SG, Fig. 9; $81 \%$ of $15.49 \mathrm{~mm}$ for MW). Based on the SG and MW frequency maps (Fig. 5), the point $60 \%$ of OC is innervated by the region of SG located

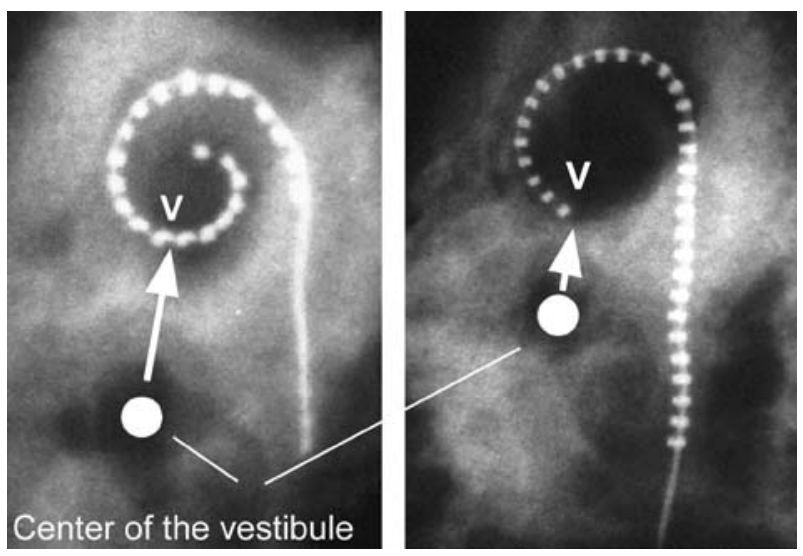

FIG. 10. An anatomical landmark $V$ in our cochlear specimens was defined as the point on the upper basal turn closest to the center of the vestibule. X-ray images here show implanted contour and banded arrays, with the electrode nearest $\mathrm{V}$ indicated. The distance required to reach $V(\sim 1,000 \mathrm{~Hz}$ for both OC and SG) for an electrode positioned under the $\mathrm{OC}$ is about $20 \mathrm{~mm}$, whereas, for an ideally positioned perimodiolar array, this distance would be only about $12-13 \mathrm{~mm}$ in the average cochlea.

at $\sim 80 \%$ from the base of the cochlea or $\sim 77 \%$ of the adjacent modiolus wall length; thus, the OC and SG frequency maps are slightly offset at this location and cochlear place frequency for the SG at $\mathrm{V}$ would be close to $800 \mathrm{~Hz}$ in the average cochlea.

\section{Frequency-position map of human SG}

Our study represents the first attempt to match frequencies along $\mathrm{OC}$ with the corresponding frequencies along the SG and describes the frequency map of the dendrites of the SG neurons as they enter Rosenthal's canal. It is possible that the frequency map of the cell somata in the extreme apex may be more offset if the radial nerve fibers continue their spiral course inside Rosenthal's canal before they reach the cell body (although we found no evidence for this). In the human cochlea, the radial nerve fibers are organized into distinct fascicles as they enter Rosenthal's canal. Each fascicle contains fibers from approximately $0.3-0.5 \mathrm{~mm}$ of OC distance. Thus, a decrease of frequency resolution due to the compression of the SG starts at the level of radial nerve fibers and may limit pitch discrimination when a CI is positioned under the well-separated fascicles. The location along the OC where fibers are no longer radial occurs at a frequency about $880 \mathrm{~Hz}$ (range of 632 to $1,055 \mathrm{~Hz}$ ). Apical to this location, the fibers have rather complex relationships with the corresponding SG cells in Rosenthal's canal, as indicated by the fact that radial nerve fibers from the apical $40 \%$ of the OC (approximately $13 \mathrm{~mm}$ ) are compressed into the apical 20\% of the SG (approximately $2.5 \mathrm{~mm}$ ). One potentially important implica- 
tion of these findings is that if the goal is to provide equal frequency resolution along CI electrode arrays, individual electrodes in perimodiolar implants should not be spaced at uniform intervals. Instead, apical electrodes would have to be more closely spaced and more basal electrodes should be more widely separated. Most contemporary electrodes, with the exception of the Contour Advance ${ }^{\mathrm{TM}}$ device, have uniformly spaced contacts. However, it must be emphasized that electrode spacing is an extremely complex issue and many other factors must be considered in defining the optimum electrode spacing. Many investigators have noted extensive channel interactions in CI users (Cohen et al. 2003; Stickney et al. 2006), and closer electrode spacing might exacerbate this problem. Computer modeling studies also suggest that dynamic range and spatial selectivity for apical electrodes (1.5 cochlear turns) are often limited due to cross-turn stimulation (Briaire and Frijns 2006). Electric field characteristics will also depend on other variables, such as array encapsulation by connective tissue, formation of new bone, and trauma to cochlear structures (Tykocinski et al. 2000; Richter et al. 2001). Computer models of the electrically stimulated cochlea should be useful in understanding the anisotropic current spread within the cochlea (Finley et al. 1990; Frijns et al. 1995, 2001; Mino et al. 2004; Briaire and Frijns 2005), and the data presented here should be valuable for more accurately specifying anatomical details for these models.

\section{Basal turn diameter measurements}

Knowledge of the individual OC and SG lengths is an important issue in estimating intracochlear position of individual electrodes. Even high-resolution CT does not allow direct measurement of the SG length, suggesting that another estimate of the total lengths of these structures would be useful. Our data indicate that a measure of overall cochlear size (e.g., the maximum diameter of the basal turn measured at the round window) as defined in our histological preparations is significantly correlated with $\mathrm{OC}$ and SG lengths. This is not surprising because, for the Archimedean spiral (the closest fit to the human cochlea), the radius of curvature is correlated to the overall length of the spiral (Ketten et al. 1998; Yoo et al. 2000).

The values reported for the basal turn diameter are in a good agreement with measurements made in CT scans in living subjects, as well as in histological preparations. Skinner et al. (2002) reported a mean value of $8 \mathrm{~mm}$ for the basal diameter in their CT images. The values for transverse cochlear diameter (close to our basal turn diameter definition of maximum diameter from the center of the round window) reported by Dimopoulos et al. (1990) for 95 temporal bone specimens had a range of 7.0 to $9.8 \mathrm{~mm}$, with a mean of $8.58 \mathrm{~mm}$. The slightly smaller values in our study (mean value $7.43 \mathrm{~mm}$ ) are likely due to the fact that our measurements were made for the OC, rather than for the outer bony wall of the cochlea.

Because basal turn diameter can be measured in imaging studies of living CI recipients, the finding of a strong correlation between basal turn diameters and OC and SG length suggests a potential method of estimating OC and SG lengths in CI users. Moreover, using the basal coil metric to predict overall OC or SG and the data shown in Figure 8 (millimeters required to achieve specific angles of rotation in cochleae of different sizes) may provide a practical means of estimating the optimum insertion length for CI electrodes in individual subjects based upon preoperative imaging studies.

\section{Electrode insertion distances and SG and OC place frequencies vs angle of rotation}

Recent studies have suggested the importance of accurate measures of intracochlear position of individual electrodes and estimates of their characteristic frequencies in understanding variability in speech recognition, improving array insertion procedures, and designing new electrodes. Several studies have shown that estimates of intracochlear electrode positions based on analysis of either 2D radiographs or CT scans are more accurate than those based on absolute insertion depth, as reported during surgery (Marsh et al. 1993; Cohen et al. 1996; Xu et al. 2000). Furthermore, Boex et al. found that using rotational insertion angles, rather than absolute insertion depths, to estimate electrode frequencies resulted in less discrepancy between pitch sensations and the frequencies predicted by Greenwood's function. However, pitch estimates were still one octave lower than predicted for OC and SG even when rotational angles were used. The authors suggested that this disparity was likely due to individual differences in OC length and imprecise estimates of the round window position on cochlear radiographs (Boex et al. 2006). Discrepancies (both lower and higher) between the frequency matched with the pitch of the electrode and the frequency predicted by intracochlear position were also reported by Baumann and Nobbe (2006).

In this study, we investigated how individual cochlear length will affect intrascalar electrode positions and the related frequency range for specific rotational angles along OC and SG. Our data showed that length of the OC is systematically related to the distance in millimeters from the base required to reach a given frequency. Thus, longer cochleae require systematically longer distances to reach a specific angle of rotation around the cochlear spiral 
as compared to the shorter specimens. This finding is in agreement with the data showing the correlation between the basal turn diameter and cochlear length. Larger cochlear diameter compensates for the increasing length of the $\mathrm{OC}$ and results in the number of turns remaining relatively constant. All of our specimens had 2.5-2.75 coils in spite of the large intersubject variation in OC length (difference of $6.37 \mathrm{~mm}$ between the shortest and longest cochleae). These data suggest that the same insertion depth in millimeters with the same electrode position relative to the inner or outer wall of the scala tympani will result in a significant range in the angular position of the contacts in different-sized cochleae, potentially reaching different characteristic frequencies. In fact, a significant correlation between the diameter of the basal turn defined on the CT images and angle of the maximum insertion depth of the electrode was found by Escude et al. (2006). This issue becomes increasingly important as more patients with residual hearing are receiving CIs, and it is essential to know what depth of insertion will be safe in preserving low-frequency hearing.

Measurements of the percentage distance of the OC and SG lengths required to reach specific angles of rotation along the cochlea showed significantly less intersubject variability than the absolute distances in millimeters. For example, the absolute difference between the shortest and longest cochleae in the distance required to reach the angle of $360^{\circ}$ from the round window is $3 \mathrm{~mm}$. If we assume an average $\mathrm{OC}$ length of about $33 \mathrm{~mm}$, this difference is approximately $10 \%$ of the OC length. At the same time, percentage distances at $360^{\circ}$ of rotation for the two extreme cochleae differ by only $5 \%$, thus suggesting a smaller extent of variability in represented frequency. At more apical locations, the difference in absolute distances (in millimeters) between the longest and shortest cochleae increases progressively, reaching 5.5 $\mathrm{mm}$ at $720^{\circ}$, which gives us $16.7 \%$ difference. In contrast, percentage distance at this angle was about $87 \%$ of total length for both the longest and shortest cochleae, with the range for the entire set being about $6 \%$. Because Greenwood's equation assigns characteristic frequencies based upon proportional distances along the $\mathrm{OC}$, the limited variability in these data suggests that frequency vs angle of rotation estimates may be relatively constant in cochleae of any size as long as adequate fixed rotational reference points (e.g., $0^{\circ}$ at the round window) are available.

\section{ACKNOWLEDGEMENTS}

This work was supported by the US National Institutes of Health, National Institute on Deafness and Other Communication Disorders, contract N01-DC-3-1006, Hearing
Research Inc. Divya Sridhar was supported by a Doris Duke Clinical Research Fellowship. The authors would like to thank Donald Greenwood, Ph.D., for his critical reading of the manuscript and valuable comments.

\section{REFERENCES}

Baskent D, Shannon R. Speech recognition under conditions of frequency-place compression and expansion. J. Acoust. Soc. Am. 113:2064-2076, 2003.

Baskent D, Shannon R. Frequency-place compression and expansion in cochlear implant listeners. J. Acoust. Soc. Am. 116(5):3130-3140, 2004.

Baumann U, Nobbe A. The cochlear implant electrode-pitch function. Hear. Res. 213(1-2):34-42, 2006.

Blamey PJ, Dooley GJ, Parisi ES, Clark GM. Pitch comparisons of acoustically and electrically evoked auditory sensations. Hear. Res. 99(1-2):139-150, 1996.

Boex C, Baud L, Cosendai G, Sigrist A, Kos Mi, Pelizzone M. Acoustic to electric pitch comparisons in cochlear implant subjects with residual hearing. J. Assoc. Res. Otolaryngol. 7:110-124, 2006.

BredBerg G. Cellular pattern and nerve supply of the human organ of Corti. Acta Otolaryngol. Suppl. 236:1-135, 1968.

BriaIRE JJ, FrIJNS JHM. Unraveling the electrically evoked compound action potential. Hear. Res. 205(1-2):143-156, 2005.

Briaire JJ, Frijns JHM. The consequences of neural degeneration regarding optimal cochlear implant position in scala tympani: a model approach. Hear. Res. 214(1-2):17-27, 2006.

Cohen LT, Xu J, Xu SA, Clark GM. Improved and simplified methods for specifying positions of the electrode bands of a cochlear implant array. Am. J. Otol. 17(6):859-865, 1996.

Cohen LT, Richardson LM, SAunders E, Cowan RSC. Spatial spread of neural excitation in cochlear implant recipients: comparison of improved ECAP method and psychophysical forward masking. Hear. Res. 179:72-87, 2003.

Dimopoulos P, Muren C. Anatomic variations of the cochlea and relations to other temporal bone structures. Acta Radiol. 31:439-444, 1990.

Dorman MF, Loizou PC, Rainey D. Simulating the effect of cochlear-implant electrode insertion depth on speech understanding. J. Acoust. Soc. Am. 102:2993-2996, 1997.

Escude B, James C, Deguine O, Cochard N, Eter E, Fraysse B. The size of the cochlea and prediction of the insertion depth angles for cochlear implant electrodes. Audiol. Neurootol. 11(Suppl):27-33, 2006.

Fayad J, Linthicum FH, Galey FR, Otto S, House W. Cochlear implants: histopathologic findings related to performance in 16 human temporal bones. Ann. Otol. Rhinol. Laryngol. 100:807-811, 1991.

Finley CC, Blake S, Wilson S, White MW. Models of neural responsiveness to electrical stimulation. In: Miller JM and Spellman FA (eds) Models of the Electrically Stimulated Ear. New York, Springer-Verlag, 1990.

Frijns JH, de Snoo SL, Schoonhoven R. Potential distributions and neural excitation patterns in a rotationally symmetric model of the electrically stimulated cochlea. Hear. Res. 87(1-2):170-186, 1995.

Frijns JH, Briaire JJ, Grote JJ. The importance of human cochlear anatomy for the results of modiolus-hugging multichannel cochlear implants. Otol. Neurotol. 22(3):340-349, 2001.

Fu QJ, SHANNON RV. Recognition of spectrally degraded and frequency shifted vowels in acoustic and electric hearing. J. Acoust. Soc. Am. 105:1889-1900, 1999.

Fu QJ, Nogaki G, Galvin JJ. Auditory training with spectrally shifted speech: implications for cochlear implant patient auditory rehabilitation. J. Assoc. Res. Otolaryngol. 6:180-189, 2005. 
Glueckert R, Pfaller K, Kinnefors A, Rask-Andersen H, SchrottFischer A. The human spiral ganglion: new insights into ultrastructure, survival rate and implications for cochlear implants. Audiol. Neurootol. 10:258-273, 2005.

GREENWOOD DD. Critical bandwidth and the frequency coordinates of the basilar membrane. J. Acoust. Soc. Am. 33:1344-1356, 1961.

GREENWOOD DD. A cochlear frequency-position function for several species-29 years later. J. Acoust. Soc. Am. 87(6):2592-2605, 1990.

GreENwOOD DD. Critical bandwidth and consonance in relation to cochlear frequency-position coordinates. Hear. Res. 54:164-208, 1991.

Hardy M. The length of the organ of Corti in man. Am. J. Anat. 62:291-311, 1968.

Hinojosa R, Marion M. Histopathology of profound sensorineural deafness. Ann. N. Y. Acad. Sci. 405:459-483, 1983.

Hochmair I, Arnold W, Nopp P, Jolly C, Muller J, Roland P. Deep electrode insertion in cochlear implants: apical morphology, electrodes and speech perception results. Acta Otolaryngol. 123:612-617, 2003.

Kawano A, Seldon HL, Clark GM. Computer-aided three-dimensional reconstruction in human cochlear maps: measurement of the lengths of organ of Corti, outer wall, inner wall, and Rosenthal's canal. Ann. Otol. Rhinol. Laryngol. 105(9):701-709, 1996.

Ketten D, Skinner MW, Wand G, Vannier MW, Gates GA, Neely JG. In vivo measures of cochlear length and insertion depth of nucleus cochlear implant electrode arrays. Ann. Otol. Rhinol. Laryngol. 107:1-16, 1998.

Khan A, Handzel O, Damian D, Eddington DK, Nadol JB. Effect of cochlear implantation on residual spiral ganglion cell count as determined by comparison with the contralateral nonimplanted inner ear in humans. Ann. Otol. Rhinol. Laryngol. 114(5):381-385, 2005.

Leake P, Hradek G. Cochlear pathology of long term neomycin induced deafness in cats. Hear. Res. 33(1):11-33, 1988.

Marsh MA, Xu J, Blamey PJ, Whitford LA, Xu SA, Silverman JM, CLARK GM. Radiologic evaluation of multichannel intracochlear implant insertion depth. Am. J. Otol. 14(4):386-391, 1993.

McFadden SL, Ding D, Jiang H, Salvi RJ. Time course of efferent fiber and spiral ganglion cell degeneration following complete hair cell loss in the chinchilla. Brain Res. 997(1):40-51, 2004.

Mino H, Rubinstein JT, Miller CA, Abbas PJ. Effects of electrode-tofiber distance on temporal neural response with electrical stimulation. IEEE Trans. Biomed. Eng. 51(1):13-20, 2004.

NADOL JBJ. Degeneration of cochlear neurons as seen in the spiral ganglion of man. Hear. Res. 49(1-3):141-154, 1990.

NADOL JB. Patterns of neural degeneration in the human cochlea and auditory nerve: implications for cochlear implantation. Otolaryngol. Head Neck Surg. 117:220-228, 1997.

NAdOL JB, Young YS, GLYNn RJ. Survival of spiral ganglion cells in profound sensorineural hearing loss: implications for cochlear implantation. Ann. Otol. Rhinol. Laryngol. 98(6):411-416, 1989.

Otte J, Chile S, Schuknecht H, Kerr AG. Ganglion cell populations in normal and pathological human cochleae. Implications for cochlear implantation. Laryngoscope 88:1231-1246, 1978.

Pfingst BE, Franck KH, Xu L, Bauer EM, Zwolan TA. Effects of electrode configuration and place of stimulation on speech perception with cochlear prostheses. J. Assoc. Res. Otolaryngol. 2(2):87-103, 2001.

Richter B, Jaekel K, Aschendorff A, Marangos N, Laszig R. Cochlear structures after implantation of a perimodiolar electrode array. Laryngoscope 111(5):837-843, 2001.

Rosen S, Faulkner A, Wilkinson L. Adaptation by normal listeners to upward spectral shifts of speech: implications for cochlear implants. J. Acoust. Soc. Am. 106:3629-3636, 1999.

Sato H, Sando I, Takahashi H. Sexual dimorphism and development of the human cochlea. Computer 3-D measurement. Acta Otolaryngol. 111(6):1037-1040, 1991.

Shannon RV, Zeng FG, Wygonski J. Speech recognition with altered spectral distribution of envelope cues. J. Acoust. Soc. Am. 104:2467-2476, 1998.

Skinner MW, Ketten DR, Holden LK, Harding GW, Smith PG, Gates GA, Neely JG, Kletzker GR, Brunsden B, Blocker B. CT-derived estimation of cochlear morphology and electrode array position in relation to word recognition in Nucleus-22 recipients. J. Assoc. Res. Otolaryngol. 3:332-350, 2002.

SRidhar D, Stakhovskaya O, Leake P. A frequency-position function for the human cochlear spiral ganglion. Audiol. Neurootol. 11(Suppl):16-20, 2006.

Stickney GS, Loizou PC, Mishra LN, Assmann PF, Shannon RV, Opie JM. Effects of electrode design and configuration on channel interactions. Hear. Res. 211(1-2):33-45, 2006.

Tykocinski M, Cohen LT, Pyman BC, Roland T Jr, Treaba C, Palamara J, Dahm MC, Shepherd RK, Xu J, Cowan RS, Cohen NL, Clark GM. Comparison of electrode position in the human cochlea using various perimodiolar electrode arrays. Am. J. Otol. 21(2):205-211, 2000.

Ulehlova L, Voldrich L, JANisch R. Correlative study of sensory cell density and cochlear length in humans. Hear. Res. 28:149-151, 1987.

Wardrop P, Whinney D, Rebscher SJ, Roland JT Jr, Luxford W, LEAKE PA. A temporal bone study of insertion trauma and intracochlear position of cochlear implant electrodes. I: comparison of Nucleus banded and Nucleus Contour ${ }^{\mathrm{TM}}$ electrodes. Hear. Res. 203:54-67, 2005a.

Wardrop P, Whinney D, Rebscher SJ, Luxford W, Leake PA. A temporal bone study of insertion trauma and intracochlear position of cochlear implant electrodes. II: comparison of Spiral Clarion ${ }^{\mathrm{TM}}$ and HiFocus II ${ }^{\mathrm{TM}}$ electrodes. Hear. Res. 203:68-79, 2005b.

Wright A, Davis A, Bredberg G, Ulehlova L, Spencer H. Hair cell distributions in the normal human cochlea. Acta Otolaryngol. Suppl. 444:1-48, 1987.

Xu J, Xu SA, Cohen LT, Clark GM. Cochlear view: postoperative radiography for cochlear implantation. Am. J. Otol. 21 (1):49-56, 2000.

Yoo SK, Wang G, Rubinstein JT, Vannier MW. Three-dimensional geometric modeling of the cochlea using helico-spiral approximation. IEEE Trans. Biomed. Eng. 47(10):1392-1402, 2000.

Yukawa K, Cohen L, Blamey P, Pyman B, Tungvachirakul V, O'LeARY S. Effects of insertion depth of cochlear implant electrodes upon speech perception. Audiol Neurootol. 9:163$172,2004$. 